\title{
MATHEMATICS EDUCATION: SOME ASPECTS CONNECTED TO ITS CONTENT
}

\author{
Paolo Bussotti \\ University of Udine, Italy \\ E-mail: paolo.bussotti@uniud.it
}

The literature concerning the various methods by means of which the teaching of mathematics can be developed is simply huge and is increasing more and more. Several aspects are dealt with: the use of new technologies, especially as far as new computer programs or web sources are concerned; new techniques to develop calculations; researches concerning the possible relations between the everyday life of the pupils/students and the mathematical concepts; the best way to frame a lesson (frontal lessons, interactive lessons, discussions), and so on. This literature covers the entire school-life of a young boy/girl: from the elementary school to the university.

If the methodological literature is abundant, the reflection on the content of mathematical teaching is inadequate. In Italy, looking at most of the handbooks for middle and high schools, one sees that the content of such texts is progressively increasing, but the logical line according to which this increment takes place is difficult to grasp. Beyond the classical subjects like algebra, analytic geometry, trigonometry, mathematical analysis, a series of new subjects is added: statistics, probability calculation, elements of linear algebra connected to some geometrical transformations (isometries, similarities, and so on), actuarial mathematics, elements of computer programming, etc. In other Western countries, the situation is not sensibly different as in Italy. Obviously, there are important differences among the different schools because the kind of mathematics taught in a classical school is not the same as the mathematics taught in a technical or professional school. However, the picture seems clear: the pupils have to learn as many subjects as possible with the risk that no subject is tackled in a profound manner, that is, a smattering of many mathematics' branches is proposed, and it is often presented in a rather superficial manner. Actually, it is evident that the role of the teacher is more important than the one of the handbooks, so that a good teacher will teach in a good manner. Nonetheless, the way in which the handbooks are conceived is a significant indication of the way in which mathematics teaching and learning is conceived.

The question to pose is: what does the school-system request from mathematics' teaching? Several answers are possible. For example:

a) Teaching to develop quickly and mechanically a series of operations (of course, not restricting to the four elementary operations);

b) teaching to win the international competitions and/or texts for mathematics;

c) teaching to develop practical abilities (for example, to collect statistical data or to program a computer), potentially useful for a future job;

d) teaching what a formal system is and how it works.

All these options are valid, and it would be a mistake to underestimate their importance.

Nevertheless, in my opinion, there is an aim which mathematics education cannot avoid: to teach how to reason in a rigorous and at the same time creative manner. It is obvious that the meaning of what is written in italics should be specified, but, for the purpose of this editorial, it is sufficient to ascribe this expression the intuitive meaning, without entering linguistic and philosophical discussions. Moreover, I give for granted that, even considering jointly the issues 
Paolo BUSSOTTI. Mathematics education: Some aspects connected to its content

PROBLEMS

OF EDUCATION

IN THE $21^{\text {st }}$ CENTURY Vol. 75, No. 6,2017

504

a)-d), the desired aim cannot be reached. This might be argued by following a series of rather easy argumentative steps.

The further question is: does a mathematical discipline exist, which might be suitable to reach the explained purpose? The answer is affirmative: such a discipline is the Euclidean geometry presented by means of the synthetic, not the analytical (in modern terms), method. Let us briefly show its richness.

Euclidean geometry is founded on five axioms or postulates:

1. A straight line segment can be drawn joining any two points.

2. Any straight line segment can be extended indefinitely.

3. Given any straight line segment, a circle can be traced having the segment as radius and one endpoint as centre.

4. All right angles are equal.

5. If two lines are traced, which intersect a third, so that the sum of the inner angles on one side is less than two right angles, then the two lines intersect each other on that side.

In a didactical presentation, it is recommended to replace this postulate with the logically equivalent, but more intuitive one:

5'. Given a straight line and a point external to such a line, it is possible to trace one and only one parallel to the given line through the given point.

These postulates are intuitive enough to be introduced when the boys/girls are 13-14.

The commentary on the postulates reveals particularly interesting aspects, which should be presented to the class.

A) The postulate 4 implies two properties: i) the measure of a right angle does not depend on the measure of its sides; ii) a right angle is not modified if it is translated or rotated. That is: the Euclidean space is homogeneous and isotropic (the teacher should explain these two concepts).

B) Then postulate 5 implies an infinitary process (this is, in part, true for the postulate 2 , as well). Under this respect, it is different from the others. Such a difference should be discussed and explained.

C) The postulates are connected to a series of operations, which they permit. To be more precise: they allow only to trace line and circles. This means that they represent all and only the operations, which are possible using a not-graduated ruler and a compass.

D) The axioms construct a world: the Euclidean world. Therefore, no proposition, no matter how simple and intuitive it might appear, can be accepted unless it derives from the axioms or from propositions derived by means of the axioms.

E) The previous issues drive to another question: Euclid's axioms are intuitive, but is it so necessary that a system of axioms is intuitive to be mathematically acceptable?

The five items A)-E) are only some of the possible conceptual and formative questions connected to the axioms, with which a teacher might deal.

After the axioms, the propositions of the first book begin. These are the basic statements of Euclidean geometry, in which, from the beginning, the necessity of rigour and creativity clearly transpires. For, there is not any a priori method, which allows the learner to solve the problem. Every problem needs a new idea. There are some guidelines, so that if one has solved many problems or proved many theorems, is accustomed with a series of procedures utilizable in Euclidean geometry. However, there is no guarantee a priori that a procedure useful to solve a problem is useful for an apparently similar problem. At the same time, each proof or construction has to be rigorous. It is not permitted to introduce surreptitiously unproved elements. I offer only one example, concerning the proposition 2 of the first book: 
Proposition 1.2.: "To place a straight-line equal to a given straight-line at a given point (as an extremity)".

Proof: Let $A$ be the given point, and $B C$ the given straight-line. So, it is required to place a straight-line at point $A$ equal to the given straight-line $B C$. For let the straight-line $A B$ have been joined from point $A$ to point $B$ [Post. 1], and let the equilateral triangle $D A B$ have been constructed upon it [Prop. 1.1]. And let the straight-lines $A E$ and $B F$ have been produced in a straight-line with $D A$ and $D B$ (respectively) [Post. 2]. And let the circle $C G H$ with centre $B$ and radius $B C$ have been drawn [Post. 3], and again let the circle $G K L$ with centre $D$ and radius $D G$ have been drawn [Post. 3]. Therefore, since the point $B$ is the centre of (the circle) $C G H, B C$ is equal to $B G$ [Def. 1.15]. Again, since the point $\mathrm{D}$ is the centre of the circle $G K L, D L$ is equal to DG [Def. 1.15]. And within these, $D A$ is equal to $D B$. Thus, the remainder $A L$ is equal to the remainder $B G$ [C.N. 3]. But $B C$ was also shown (to be) equal to $B G$. Thus, $A L$ and $B C$ are each equal to $B G$. But things equal to the same thing are also equal to one another [C.N. 1]. Thus, $A L$ is also equal to $B C$. Thus, the straight-line $A L$, equal to the given straight-line $B C$, has been placed at the given point $A$. (Which is) the very thing it was required to do (Euclid 2007-2008, pp. 8-9).

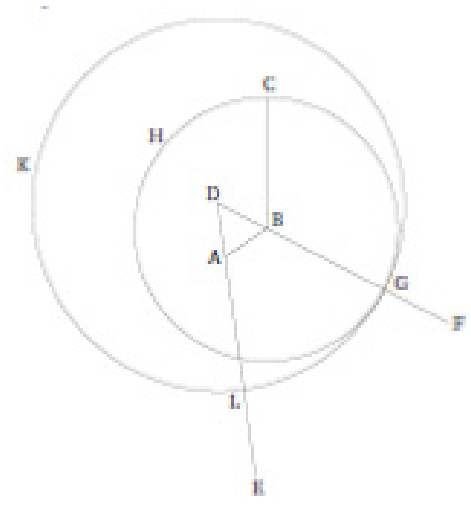

Figure 1: The figure added by Euclid to prove Proposition 1.2. Retrieved from Euclid's Elements of Geometry, 2007-2008, p. 9.

The proof of this easy proposition makes it clear what I mean: rigour, no unproved or unjustified element is introduced. In order: Postulate 1; Proposition 1.1; Postulate 2; Postulate 3 , twice; Definition 1.15 (this is a part of the text immediately preceding the axioms); Common Notion 3, twice (this is a part of the text immediately following the postulates); Common Notion 1 is used to support the foundations of the proof.

At the same time, the notion of construct of the two circles is not pre-ordered, it is necessary to develop creativity, to have a little brilliant idea.

In the course of the scholastic years an itinerary along Euclid's masterpiece might be developed. As to the first book, it could be possible to carry out the problem of triangles' criteria of congruence, of the use, starting from Proposition 1.29, of the fifth postulate. In this important conceptual and historical case, some lessons might be conceived on the nature of this postulate. A first, initial introduction to the not-Euclidean geometries might be outlined. The first book is closed by the so-called Pythagorean Theorem and its inverse proposition (1.47 and 1.48 respectively). In this case, too, a series of lessons could be dedicated to this important proposition and its applications. The second book is brief and easy. It concerns the so told geometrical algebra. The third and the fourth books, which are relative to the properties of the circle are as important and formative as the first one.

With the fifth Element's book, the Euclidean geometry, makes a breakthrough: the theory of proportions is introduced with all the nuances and operations connoting this only apparently easy concept. In the sixth book, the theory of proportion is applied to geometry and the theory 
Paolo BUSSOTTI. Mathematics education: Some aspects connected to its content

PROBLEMS

OF EDUCATION

IN THE $21^{\text {st }}$ CENTURY Vol. 75, No. 6,2017

506

of similarity is presented. This subject is ideal to be presented in the third and fourth year of the high school. The most difficult and fascinating Euclidean problems, which in most cases are not present in the Elements, but have been conceived, argued and proved in the course of history of mathematics, rely upon the concept of similarity and on the criteria established in the sixth book. It is not by chance that Felix Klein has proved, within group theory, that the Euclidean geometry is the group of the similarities (Klein, 1872, 1893). As to the content, the books VII$\mathrm{X}$ might be bypassed. Albeit some elements of the theory of irrationalities are necessary, it is not needed, in this case, to follow Euclid's approach. The last year of the high school should be devoted to the books XI-XIII: solid geometry.

Along the didactical iter based on Euclidean geometry, important logical and conceptual questions might be dealt with: the nature of the ad absurdum demonstration; the concept of existence in mathematics; the difference between a synthetic and an analytical ( $a$ là Greek) approach, the use of the figures within a mathematical demonstration, only to mention the most stimulating subjects.

I do not necessarily mean that Euclid's text should be followed line by line. Some very good handbooks exist. As far as I know, one of the best is Enriques-Amaldi 1903, with several reprints, translations (Spanish, Polish), and updating (this book also presents a huge series of exercises). However, good handbooks exist in any country. What is important is that the Euclidean geometry comes back to be one of the main (obviously, not the only one) mathematical disciplines taught in our schools (Bussotti, 2012a, Bussotti 2012b, Bussotti, 2013, Russo, Pirro, Salciccia, 2017). The reasons are those expounded.

Some aspects of Euclidean geometry are taught nowadays, but they are so marginal, are not inserted within an organic program and are taught without the necessary rigour that their influence on the school-background of a young is almost insignificant. After the school, when the young attend the university, if they frequent scientific faculties, a completely different approach than Euclid's is developed: an instrumental and, so to say, practical one, if they attend Engineering and, in part, Physics, a very abstract one, if they attend Mathematics. For the students, who do not frequent such faculties, the mathematics examinations are few in numbers, and, certainly, they are not dedicated to the synthetic geometry. In a sense, the faculties of Architecture, with the teaching of Descriptive Geometry are those where, at least a limited number of proofs has some aspects comparable with those of the Euclidean geometry.

It is my conviction that Euclidean Geometry should be the basis of mathematics education and that the students who desire to become teachers of mathematics should sustain two examinations on Euclidean geometry.

\section{References}

Bussotti, P. (2012a). History and didactics of mathematics: a problematic relation. Some considerations based on Federigo Enriques's ideas. Problems of Education in the 21st Century, 48, 5-9.

Bussotti, P. (2012b). Federigo Enriques e la didattica della matematica [Federigo Enriques and mathematics education]. Euclide. Giornale di matematica per $i$ giovani, Electronic journal. First part February 2012, second part April 2012. web site: http://www.euclide-scuola.org/.

Bussotti, P. (2013). A possible role fo history of mathematics and science in mathematics and science education. Journal of Baltic Science Education, 12 (6), 712-715.

Enriques, F., Amaldi, U. (1903). Elementi di geometria ad uso delle scuole secondarie superiori [Elements of geometry for high schools]. Bologna: Zanichelli.

Euclid (2007-2008). Elements of Geometry, The Greek text of J.L. Heiberg (1883-1885)from Euclidis Elementa, edidit et Latine interpretatus est I. L. Heiberg, in aedibus B.G. Teubneri, 1883-1885. Edited and provided with a modern English translation by Richard Fitzpatrick. Retrieved from http://farside.ph.utexas.edu/Books/Euclid/Elements.pdf. 
Klein, F. (1872, 1893). A comparative review of recent researches in geometry (Programme on entering the philosophical faculty and the senate of the university of Erlangen in 1872). Bulletin of the New York Mathematical Society, 2, 215-249.

Russo, L., Pirro, G., Salciccia, E. (2017). Euclide: il I libro degli Elementi [Euclid: the first book of Elements]. Roma: Carocci Editore.

Received: December 05, 2017

Accepted: December 10, 2017

OF EDUCATION

IN THE $21^{\text {st }}$ CENTURY

Vol. 75 , No. 6, 2017

507

Paolo Bussotti

$\mathrm{PhD}$, Researcher and Lecturer of History of Science and History of Mathematics, via Petracco and via delle scienze, University of Udine, Italy.

E-mail: paolo.bussotti@uniud.it

Website: https://www.researchgate.net/profile/Paolo_Bussotti 